\title{
Matter vs. vacuum oscillations at long-baseline accelerator neutrino experiments
}

\author{
Suman Bharti, ${ }^{1, *}$ Ushak Rahaman, $^{2, \dagger}$ and S. Uma Sankar ${ }^{1, \ddagger}$ \\ ${ }^{1}$ Department of Physics, Indian Institute of Technology Bombay, Mumbai 400076, India \\ ${ }^{2}$ Centre for Astro-Particle Physics (CAPP) and Department of Physics, \\ University of Johannesburg, PO Box 524, Auckland Park 2006, South Africa
}

(Dated: October 6, 2020)

\begin{abstract}
The neutrino oscillation probabilities at the long-baseline accelerator neutrino experiments are expected to be modified by matter effects. We search for evidence of such modification in the data of $\mathrm{T} 2 \mathrm{~K}$ and $\mathrm{NO} \nu \mathrm{A}$, by fitting the data to the hypothesis of (a) matter modified oscillations and (b) vacuum oscillations. We find that vacuum oscillations provide as good a fit to the data as matter modified oscillations. Even extended runs of $\mathrm{T} 2 \mathrm{~K}$ and $\mathrm{NO} \nu \mathrm{A}$, with 5 years in neutrino mode $(5 \nu)$ and five years in anti-neutrino mode $(5 \bar{\nu})$, can not make a $3 \sigma$ distinction between vacuum and matter modified oscillations. The proposed experiment DUNE, with neutrino and anti-neutrino runs of 5 years each $(5 \nu+5 \bar{\nu})$, can rule out vacuum oscillations by itself at $5 \sigma$ if the hierarchy is normal. If the hierarchy is inverted, a $5 \sigma$ discrimination against vacuum oscillations requires the combination of $(5 \nu+5 \bar{\nu})$ runs of $\mathrm{T} 2 \mathrm{~K}, \mathrm{NO} \nu \mathrm{A}$ and DUNE.

PACS numbers: 14.60.Pq,14.60.Lm,13.15.+g
\end{abstract}

Keywords: Neutrino Mass Hierarchy, Long-Baseline Experiments

\footnotetext{
* Email Address: sbharti@phy.iitb.ac.in

† Email Address: ushakr@uj.ac.za

‡ Email Address: uma@phy.iitb.ac.in
} 


\section{INTRODUCTION}

Neutrino oscillations provide a signal for physics beyond standard model. Over the past 20 years, a large number of experiments have determined neutrino oscillation parameters to better and better precision. Of these, there are two different types of oscillations, one driven by a smaller mass-squared difference (labelled $\Delta_{21}$ by convention) and another driven by a larger mass-squared difference (labelled $\Delta_{31}$ by convention). The first hint of the oscillations, driven by the larger mass-squared difference $\Delta_{31}$, was noted in the deficit of upgoing atmospheric $\nu_{\mu} / \bar{\nu}_{\mu}$ events by the pioneering water Cerenkov detectors IMB [1, 2] and Kamiokande $[3,4]$. The atmospheric neutrino experiment Super-Kamiokande [5] and the long-baseline accelerator neutrino experiments MINOS [6], T2K [7] and NO $\nu \mathrm{A}$ [8] observed the spectral distortions in the survival probabilities of $\nu_{\mu}$ and $\bar{\nu}_{\mu}$. Initial analysis of these distortions was done under the hypothesis of vacuum oscillations and the values of the oscillation parameters $\left|\Delta_{31}\right|$ and $\sin 2 \theta_{23}$ were obtained. These experiments could not distinguish between positive and negative values of $\Delta_{31}$. The case of positive $\Delta_{31}$ is called normal hierarchy $(\mathrm{NH})$ and that of negative $\Delta_{31}$ is called inverted hierarchy (IH). For $\sin 2 \theta_{23}<1$, the same value is realized by two different values of $\theta_{23}$, one $<45^{\circ}$ in the lower octant (LO) and the other $>45^{\circ}$ in the higher octant $(\mathrm{HO})$.

Due to the propagation of the neutrinos through earth matter, it is expected that the oscillation probabilities would be modified by matter effects. These matter effects are sensitive to the sign of $\Delta_{31}$ and their observation can lead to a determination of this sign. For baselines less than $1000 \mathrm{~km}$, the matter effects lead to negligibly small changes in $\nu_{\mu} / \bar{\nu}_{\mu}$ survival probabilities [9]. Thus, the $\nu_{\mu} / \bar{\nu}_{\mu}$ disappearance data of accelerator neutrino experiments lead to essentially the same values of $\left|\Delta_{31}\right|$ and $\sin 2 \theta_{23}$ for the three cases: (a) vacuum oscillations, (b) matter oscillations with $\mathrm{NH}$ and (c) matter oscillations with IH. In the case of atmospheric neutrinos, the survival probabilities $P_{\mu \mu}$ and $P_{\bar{\mu} \bar{\mu}}$ are expected to undergo significant changes due to matter effects. However, at present Super-Kamiokande is able to make only a small distinction between them [10]. A number of studies [9, 11-13] explored the sensitivity of future atmospheric neutrino detectors to observe these matter effects and determine whether $\Delta_{31}$ is positive or negative (or whether hierearchy is $\mathrm{NH}$ or $\mathrm{IH})$.

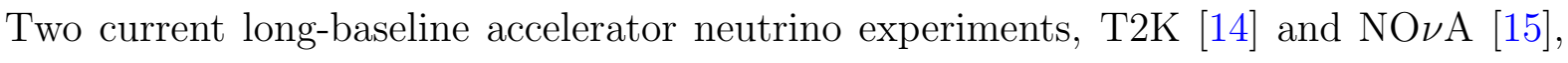


are looking for evidence of the matter modification of oscillation probabilities. These experiments measure the survival probabilities, $P\left(\nu_{\mu} \rightarrow \nu_{\mu}\right)$ and $P\left(\bar{\nu}_{\mu} \rightarrow \bar{\nu}_{\mu}\right)$, and the oscillation probabilities, $P\left(\nu_{\mu} \rightarrow \nu_{e}\right)$ and $P\left(\bar{\nu}_{\mu} \rightarrow \bar{\nu}_{e}\right)$. For baselines of the order of $1000 \mathrm{~km}$ or less, the changes in survival probabilities are negligibly small but the changes in the oscillation probabilities are noticeable. Hence the ability of these two experiments to search for the matter effects comes essentially from the measurement of $P\left(\nu_{\mu} \rightarrow \nu_{e}\right)$ and $P\left(\bar{\nu}_{\mu} \rightarrow \bar{\nu}_{e}\right)[16,17]$. But these appearance probabilities are also sensitive to the unknown $\mathrm{CP}$ violating phase $\delta_{\mathrm{CP}}$. Given a set of data, three solutions are likely to occur [18, 19]:

- matter modified oscillations with $\mathrm{NH}$ and $\delta_{\mathrm{CP}}^{1}$,

- vacuum oscillations and $\delta_{\mathrm{CP}}^{2}$ and

- matter modified oscillations with IH and $\delta_{\mathrm{CP}}^{3}$.

For $\mathrm{NO} \nu \mathrm{A}$, the changes induced in $P_{\mu e}$ and in $P_{\bar{\mu} \bar{e}}$ due to matter effects are comparable to the changes induced when the value of $\delta_{\mathrm{CP}}$ is changed by $90^{\circ}$ [20]. Hence, the measured value of $\delta_{\mathrm{CP}}$ depends significantly on the oscillation hypothesis that is used. For T2K experiment, the matter effects lead to a smaller change in the appearance probabilities. Hence the value of $\delta_{\mathrm{CP}}$ determined from T2K data is less sensitive to whether matter effects are included or not. Establishing CP violation in neutrino oscillations is one of the most important goals of both current and future long-baseline accelerator neutrino experiments. To achieve this goal, it is important to establish a distinction between vacuum oscillations and matter modified oscillations.

The matter effect is included in the neutrino evolution in the form of the Wolfenstein matter term [21]

$$
A\left(\text { in } \mathrm{eV}^{2}\right)=0.76 \times 10^{-4} \rho(\text { in gm/cc }) E(\text { in } \mathrm{GeV}),
$$

where $\rho$ is the density of matter and $E$ is the energy of neutrino. The presence of this matter term modifies both the mass-square differences and the mixing angles and hence the neutrino survival/oscillations probabilities. Matter effects play a crucial role in the solution for solar neutrino deficit [22-24]. The existence of matter effects in oscillations driven by $\Delta_{21}$ is established at a significance better than $5 \sigma$ [25]. However, as mentioned above, there is no evidence as yet for matter effects in the oscillations driven by $\Delta_{31}$. The existence of 
matter effects at this scale should be verified independently, just as the oscillations by the two different mass-squared differences are established indepedently.

The expression for matter modified $P\left(\nu_{\mu} \rightarrow \nu_{e}\right)$ for $\mathrm{T} 2 \mathrm{~K}$ and $\mathrm{NO} \nu \mathrm{A}$ experiments is given by $[26,27]$

$$
\begin{aligned}
P\left(\nu_{\mu} \rightarrow \nu_{e}\right)= & \sin ^{2} 2 \theta_{13} \sin ^{2} \theta_{23} \frac{\sin ^{2} \hat{\Delta}(1-\hat{A})}{(1-\hat{A})^{2}}+ \\
& \alpha \cos \theta_{13} \sin 2 \theta_{12} \sin 2 \theta_{13} \sin 2 \theta_{23} \cos \left(\hat{\Delta}+\delta_{\mathrm{CP}}\right) \frac{\sin \hat{\Delta} \hat{A} \sin \hat{\Delta}(1-\hat{A})}{\hat{A}} \frac{\hat{A}}{1-\hat{A}}
\end{aligned}
$$

where $\hat{\Delta}=1.27 \Delta_{31} L / E, \hat{A}=A / \Delta_{31}$ and $\alpha=\Delta_{21} / \Delta_{31}$. The expression for $P\left(\bar{\nu}_{\mu} \rightarrow \bar{\nu}_{e}\right)$ is obtained by replacing $A$ by $-A$ and $\delta_{\mathrm{CP}}$ by $-\delta_{\mathrm{CP}}$. The corresponding probabilities for vacuum oscillations can be obtained by taking the limit $A \rightarrow 0$. We note that the two appearance probabilities depend not only on the unknown hierarchy but also on the two other unknowns $\delta_{\mathrm{CP}}$ and the octant of $\theta_{23}$. Depending on the measured values of $P_{\mu e}$ and $P_{\bar{\mu} \bar{e}}$ it is possible to cancel the change induced by the matter effects by choosing a wrong value of $\delta_{\mathrm{CP}}$ and/or $\theta_{23}$ [20]. Hence establishing unambiguous evidence for matter effects at long-baseline experiments is non-trivial.

In this work we study the distinction made by the present data of $\mathrm{T} 2 \mathrm{~K}$ and $\mathrm{NO} \nu \mathrm{A}$ between vacuum oscillations and matter modified oscillations (for both hierarchies). We find that matter modified oscillations with normal hierarchy do provide the best fit solution to the data but the vacuum oscillations provide nearly as good a fit. We also consider the ability of T2K and $\mathrm{NO} \nu \mathrm{A}$ to make a distinction by the end of their runs. We find that a $3 \sigma$ discrimination is not possible even if each experiment has a $(5 \nu+5 \bar{\nu})$ run (that is, 5 years each in neutrino and anti-neutrino modes). We further study the ability of the future experiment DUNE to make such a discrimination. The baseline and hence the energy of the neutrino beam of DUNE are larger which lead to larger changes in $P\left(\nu_{\mu} \rightarrow \nu_{e}\right)$ and $P\left(\bar{\nu}_{\mu} \rightarrow \bar{\nu}_{e}\right)$ due to matter effects. A one year neutrino run of DUNE by itself can make a $3 \sigma$ discrimination between matter and vacuum oscillations, if the hierarchy is normal. Addition of the T2K and $\mathrm{NO} \nu \mathrm{A}$ data to one year of DUNE data leads only to a small improvement in this discrimination. Vacuum oscillations can be ruled out at $5 \sigma$, for both normal and inverted hierarchies, by the combined T2K $(5 \nu+5 \bar{\nu}), \mathrm{NO} \nu \mathrm{A}(5 \nu+5 \bar{\nu})$ and DUNE $(5 \nu+5 \bar{\nu})$ runs. 


\section{ANALYSIS PROCEDURE}

We use the following procedure to generate our results. We calculated the theoretical event spectra with three flavour oscillations using GLoBES [28, 29], for the appearance and disappearance channels in both neutrino and anti-neutrino modes for $\mathrm{T} 2 \mathrm{~K}$ and for $\mathrm{NO} \nu \mathrm{A}$. We have tuned the efficiencies in the software such that we get a match with the observed number of events when the best-fit oscillation parameters are used as input. These rates are calculated with the matter potential parametrized as $q * A$, where $A$ is the standard Wolfenstein matter term and $q$ is a multiplicative factor. In this analysis, we consider the possibility of non-standard matter term, as was done in ref. [10]. The following inputs are used in our calculations: the solar neutrino parameters $\sin ^{2} \theta_{12}$ and $\Delta_{21}$ were held fixed at 0.31 and $7.39 \times 10^{-5} \mathrm{eV}^{2}$ respectively. The values of $\sin ^{2} \theta_{13}$ were varied in its $3 \sigma$ range around its central value 0.02237 (0.02259) with $\sigma=0.00066$ (0.00065) for NH (IH). The values of $\sin ^{2} \theta_{23}$ were varied in its $3 \sigma$ range around its central value $0.563(0.565)$ with $\sigma=0.024(0.022)$ for $\mathrm{NH}(\mathrm{IH})$. The value of $\left|\Delta_{31}\right|$ was varied in its $3 \sigma$ range around its central value $2.528 \times 10^{-3} \mathrm{eV}^{2}\left(2.510 \times 10^{-3} \mathrm{eV}^{2}\right)$ with $\sigma=0.031 \times 10^{-3} \mathrm{eV}^{2}$ for $\mathrm{NH}(\mathrm{IH})$. The $\mathrm{CP}$ violating phase $\delta_{\mathrm{CP}}$ is varied in its full range $\left(0,360^{\circ}\right)$. The best-fit values and the $3 \sigma$ ranges of the measured neutrino oscillation parameters are taken from ref. [30, 31]. The theoretical event rates are calculated separately for both the test hierarchies, $\mathrm{NH}$ and $\mathrm{IH}$. The non-standard matter interaction parameter $q$ is varied between $(0,2)$. The value $q=0$, of course, stands for vacuum oscillations.

In the first instance, we compare these theoretical event rates with the present data of $\mathrm{T} 2 \mathrm{~K}$ and $\mathrm{NO} \nu \mathrm{A}$. This is done by computing the $\chi^{2}$ between the theory and data for each of the four data sets of each experiment. For a particular experiment and for a particular data set, the Poissonian $\chi^{2}$ is calculated using the expression

$$
\chi^{2}=\sum_{i} 2\left[\left(N_{i}^{\mathrm{th}}-N_{i}^{\mathrm{exp}}\right)+N_{i}^{\mathrm{exp}} \times \ln \left(N_{i}^{\mathrm{exp}} / N_{i}^{\mathrm{th}}\right)\right]+\sum_{j}\left[2 \times N_{j}^{\mathrm{th}}\right]+\chi^{2}(\text { sys }),
$$

where $i$ stands for bins for which $N_{i}^{\exp } \neq 0$ and $j$ stands for bins for which $N_{j}^{\text {exp }}=0$. The term $\chi^{2}$ (sys) arises due to systematic uncertainties. For each of the two experiments, we included systematic uncertainties of $10 \%$, using the pull method. We varied the pull parameter in $3 \sigma$ range and marginalized over it to determine $\chi_{m}^{2}$ as a function of test values of oscillation parameters, mass hierarchies and $q$. As explained in the introduction, the 
disappearance data in these experiments has negligible sensitivity to matter effects. The main sensitivity comes from the appearance data, which has a limited number of events because of the small value of $\theta_{13}$. The appearance events are significant in a small number of energy bins. The sensitivity to matter effects is more dependent on the total number of apperance events than on the energy distribution. The largest systematic uncertainty in this number is in the number of expected $\nu_{\mu}$ events in the case of no oscillations. We took this systematic uncertainty to be $10 \%$ and neglected the small variation across the small number of energy bins.

We calculated the total $\chi^{2}$ for both $\mathrm{NH}$ test and IH test as

$$
\begin{aligned}
\chi^{2}(\mathrm{tot})= & \chi_{m}^{2}(\mathrm{NO} \nu \mathrm{A} \nu \mathrm{app})+\chi_{m}^{2}(\mathrm{NO} \nu \mathrm{A} \bar{\nu} \mathrm{app})+\chi_{m}^{2}(\mathrm{~T} 2 \mathrm{~K} \nu \mathrm{app}) \\
& +\chi_{m}^{2}(\mathrm{~T} 2 \mathrm{~K} \bar{\nu} \mathrm{app})+\chi_{m}^{2}(\mathrm{NO} \nu \mathrm{A} \nu \text { disapp })+\chi_{m}^{2}(\mathrm{NO} \nu \mathrm{A} \bar{\nu} \operatorname{disapp}) \\
& +\chi_{m}^{2}(\mathrm{~T} 2 \mathrm{~K} \nu \text { disapp })+\chi_{m}^{2}(\mathrm{~T} 2 \mathrm{~K} \bar{\nu} \text { disapp })+\chi^{2}(\text { prior })
\end{aligned}
$$

We added priors on $\sin ^{2} \theta_{13}, \sin ^{2} \theta_{23}$ and $\left|\Delta_{31}\right|$. During the calculation of $\chi^{2}$ (tot), we have to keep in mind that the test values of the oscillation parameters are the same for all the individual $\chi^{2}$ s. This quantity $\chi^{2}$ (tot) is a function of test values of oscillation parameters, hierarchies and $q$. We found the minimum of $\chi^{2}$ (tot) and subtracted it from all other values of $\chi^{2}$ (tot) to obtain $\Delta \chi^{2}$ as a function of test values of oscillation parameters, hierarchies and $q$. At the last step, we marginalized $\Delta \chi^{2}$ over all the oscillations parameters but not over hierarchy and $q$.

In later stages we simulated the expected data from the future runs of $\mathrm{T} 2 \mathrm{~K}$ and $\mathrm{NO} \nu \mathrm{A}$ and also the runs of the future experiment DUNE. These simulations were done with the best fit values [30] of mass-squared differences, mixing angles, $\delta_{\mathrm{CP}}$ and $q=1$. These were done separately for both $\mathrm{NH}$ and for $\mathrm{IH}$ as true hierarchy. The results of these simulations were used as data and the theoretical event rates and $\chi^{2}$ were calculated as described earlier. In the case of this calculation, $\chi^{2}$ is equivalent to $\Delta \chi^{2}$.

\section{RESULTS}

\section{A. From present data}

So far $\mathrm{NO} \nu \mathrm{A}$ has taken data $8.85 \times 10^{20} \mathrm{POT}$ in $\nu$ mode and $12.33 \times 10^{20} \mathrm{POT}$ in $\bar{\nu}$ mode. The disappearance and appearance spectra for both modes are given in ref. [32]. At 
present, T2K has taken data with $14.9 \times 10^{20} \mathrm{POT}$ in $\nu$ mode and $16.4 \times 10^{20} \mathrm{POT}$ in $\bar{\nu}$ mode [33]. The appearance event spectra (for both $\nu$ and $\bar{\nu}$ modes) are given in ref. [33] but the disappearance event spectra for the full data set are not available. Ref. [34] gives the disappearance spectra for $14.7 \times 10^{20} \mathrm{POT}$ in $\nu$ mode and $7.6 \times 10^{20} \mathrm{POT}$ in $\bar{\nu}$ mode. We use the above mentioned spectra in our analysis. As explained in the introduction, the change induced in the survival probabilities due to matter effects is quite small. Therefore, we expect that our results would remain the same even when the disappearance spectra of the full $\mathrm{T} 2 \mathrm{~K}$ data are used in the analysis.

The above data set, with 152 data points, was fit to the hypothesis of three flavour oscillations with variable matter term as described in the previous section and the results are displayed in fig. 1. The minimum $\chi^{2}=173.2$ occurs for $\Delta_{31}$ positive and $q=0.7$. Standard matter oscillations with $\mathrm{NH}(q=1)$ has essentially the same $\chi^{2}$ whereas the standard matter effects with $\mathrm{IH}$ are disfavored by a $\Delta \chi^{2}=4.5$. It is interesting to note that vacuum oscillations $(q=0)$ provide nearly as good a fit to the data as matter modified oscillations with $\mathrm{NH}$ (with $\left.\chi^{2}=173.8\right)$.

To explicitly verify that the best-fit vacuum oscillations provide nearly as good a fit to the data as the best-fit matter modified oscillations, we have plotted the observed appearance event numbers in both neutrino and anti-neutrino modes for the two experiments, along with the predicted rates. The results are shown in fig. 2. From the plots in this figure, we note that there is hardly any difference between the predictions of vacuum and matter modified oscillations and each fits the data very well.

As stated in the introduction, the best-fit value of $\delta_{\mathrm{CP}}$ depends on the oscillation hypothesis that is used to fit the data. In fig. 3, we show the best-fit points and $1 \sigma$ contours of $\mathrm{T} 2 \mathrm{~K}$ data, $\mathrm{NO} \nu \mathrm{A}$ data and $\mathrm{T} 2 \mathrm{~K}+\mathrm{NO} \nu \mathrm{A}$ data for the two cases of matter modified and vacuum oscillations. Comparing the two cases, we find that the best-fit point of T2K is essentially the same for both vacuum and matter modified oscillations whereas the best-fit value of $\delta_{\mathrm{CP}}$ in the case of $\mathrm{NO} \nu \mathrm{A}$ strongly depends on the oscillation hypothesis used in the fit. It is also interesting to note that there is less discrepancy between the best-fit points of the two experiments in the case of vacuum oscillations than in the case of matter modified oscillations. 


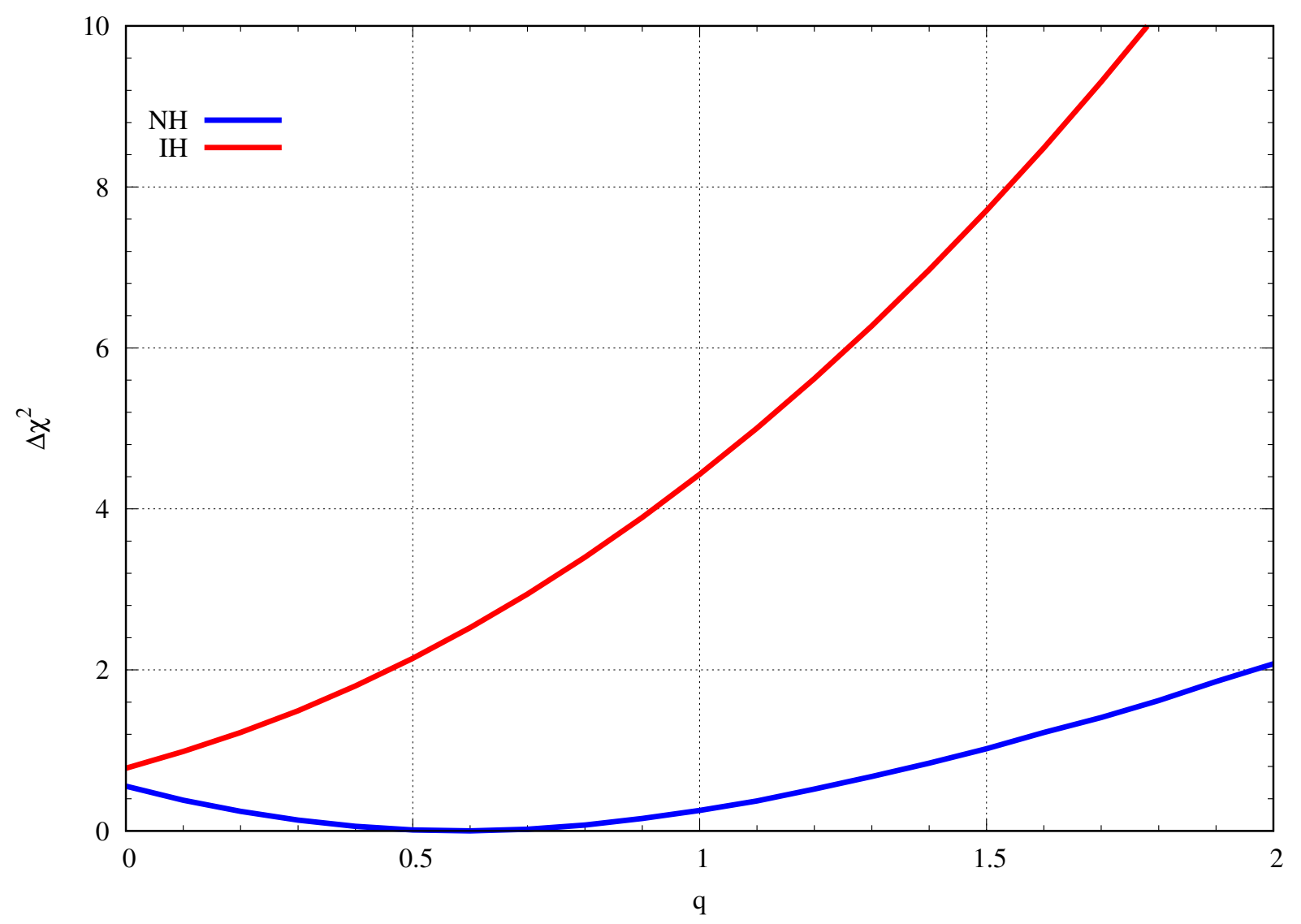

FIG. 1: $\Delta \chi^{2}$ vs $q$ for the present data of $\mathrm{T} 2 \mathrm{~K}$ and $\mathrm{NO} \nu \mathrm{A}$. The data of $\mathrm{NO} \nu \mathrm{A}$ consists of $8.85 \times 10^{20}$ POT in $\nu$ mode and $12.33 \times 10^{20} \mathrm{POT}$ in $\bar{\nu}$ mode. The appearance data of T2K consists of $14.9 \times 10^{20}$ POT in $\nu$ mode and $16.4 \times 10^{20} \mathrm{POT}$ in $\bar{\nu}$ mode. The disappearance data of T2K consists of $14.7 \times 10^{20}$ POT in $\nu$ mode and $7.6 \times 10^{20} \mathrm{POT}$ in $\bar{\nu}$ mode. The minimum $\chi^{2}$, for 152 data points is 173.2 . The blue (red) curve is for $\mathrm{NH}(\mathrm{IH})$. Note that vacuum oscillations $(q=0)$ also provide a good fit to the data.

\section{B. Expectation from extended runs of $\mathrm{T} 2 \mathrm{~K}$ and $\mathrm{NO} \nu \mathrm{A}$}

We now consider the ability of extended runs of $\mathrm{T} 2 \mathrm{~K}$ and $\mathrm{NO} \nu \mathrm{A}$ to establish matter modified oscillations. Using GLoBES, we simulated event spectra of T2K for $37.4 \times 10^{20}$ POT each in both $\nu$ and $\bar{\nu}$ mode corresponding to a five year run in each mode. The simulation for $\mathrm{NO} \nu \mathrm{A}$ was done for $30.25 \times 10^{20} \mathrm{POT}$ each in both $\nu$ and $\bar{\nu}$ mode, again corresponding to a five year run in each mode. We used the best fit values, taken from the global fits $[30,31]$, for the neutrino oscillation parameters in doing these simulations, which were done separately for NH being the true hierarchy and for IH being the true hierarchy. The simulated data is analyzed in the manner described in section 2 and the results are shown 


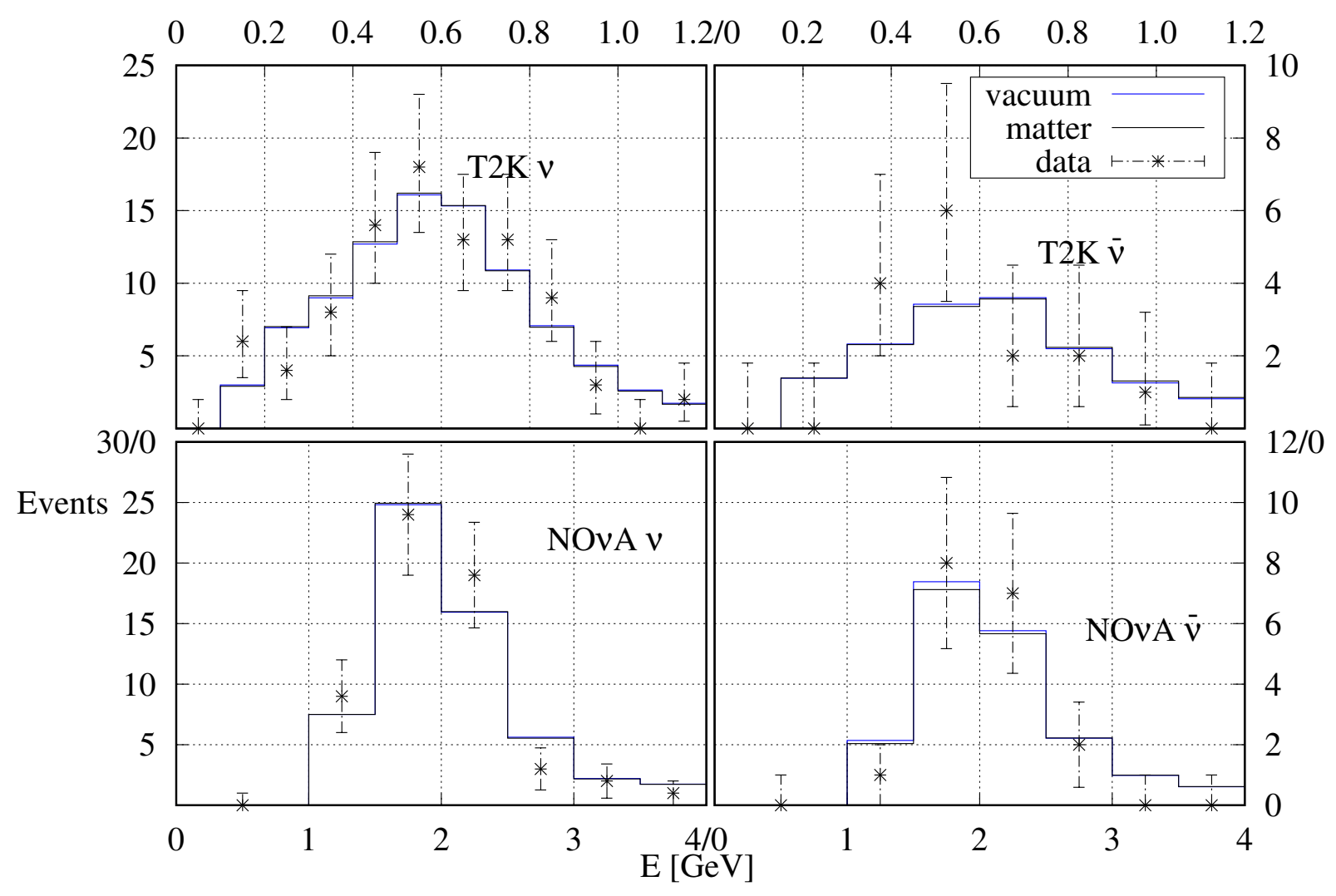

FIG. 2: The observed number of events in appearance mode (shown as stars) and the corresponding predictions from best-fit vacuum oscillations (blue histogram) and best-fit matter modified oscillations (black histogram) for $\mathrm{T} 2 \mathrm{~K}$ (upper row) and $\mathrm{NO} \nu \mathrm{A}$ (lower row). The figures in the left panels are for $\nu_{e}$ appearance and those in the right panels are for $\bar{\nu}_{e}$ appearance.

in fig. 4. We note that such an extended run rules out IH at $3 \sigma$ if $\mathrm{NH}$ is the true hierarchy but rules out $\mathrm{NH}$ only at $2 \sigma$ if $\mathrm{IH}$ is the true hierarchy. We also note that, no matter what the true hierarchy is, the vacuum oscillations have a very small $\Delta \chi^{2} \simeq 2$. Therefore the combined data of $\mathrm{T} 2 \mathrm{~K}$ and $\mathrm{NO} \nu \mathrm{A}$ cannot distinguish between matter modified oscillations and vacuum oscillations.

\section{Expectation from DUNE}

The future long-baseline accelerator neutrino experiment DUNE [35-37] is designed to disentangle the changes due to matter effects from the changes due to $\delta_{\mathrm{CP}}$. Its baseline ( $L \simeq 1300 \mathrm{~km}$ ) is much longer than that of $\mathrm{T} 2 \mathrm{~K}$ or $\mathrm{NO} \nu \mathrm{A}$. Its peak energy is correspondingly 

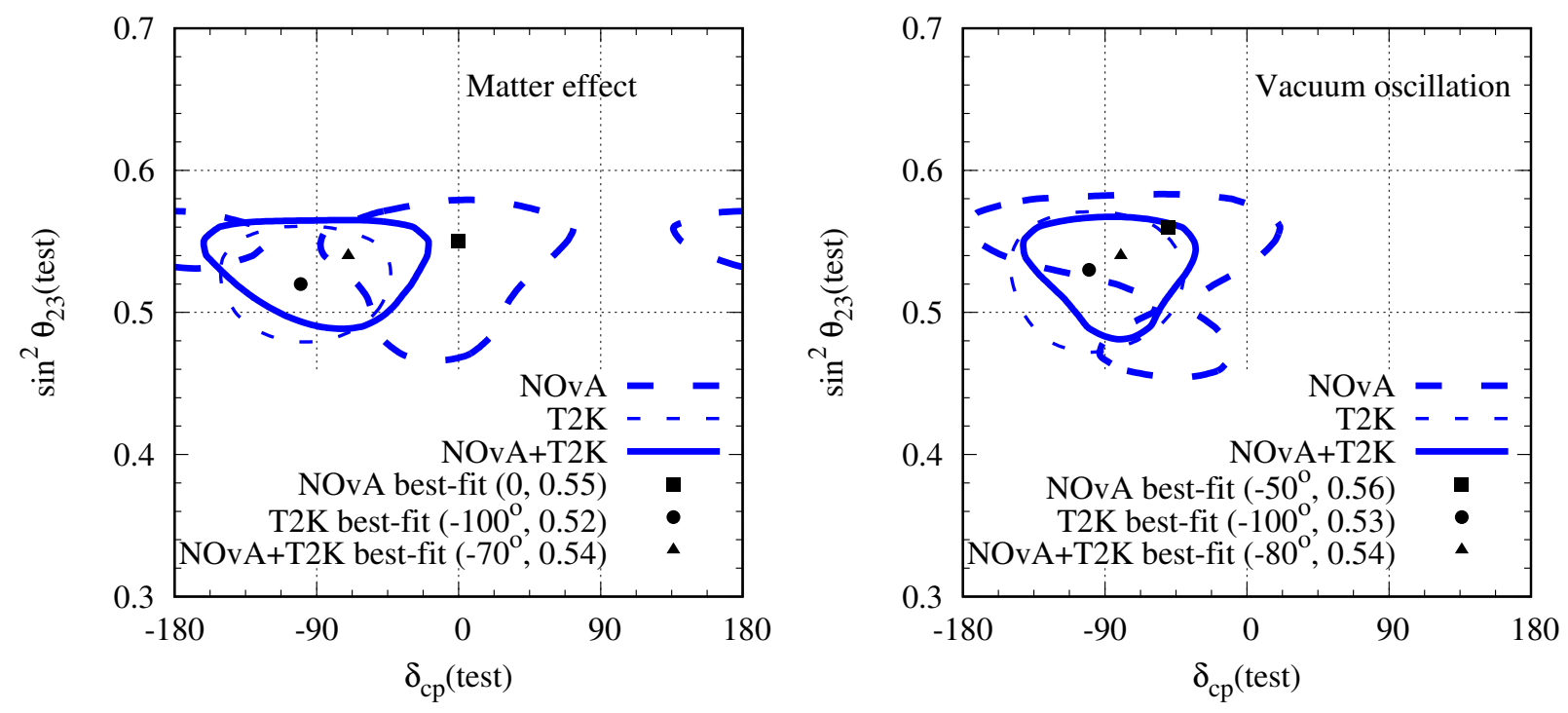

FIG. 3: The best-fit points and $1 \sigma$ contours of T2K data (thin dashed lines), NO $\nu$ A data (thick dashed lines) and $\mathrm{T} 2 \mathrm{~K}+\mathrm{NO} \nu \mathrm{A}$ data (solid lines) for the case of matter modified oscillations (left panel) and vacuum oscillations (right panel).

higher and matter effects larger. Therefore, it is expected that it will have a much better ability to rule out vacuum oscillations. Fig. 5 shows the ability of one year neutrino run of DUNE to establish matter modified oscillations. We note that vacuum oscillations are ruled out at $3 \sigma$ if the true hierarchy is $\mathrm{NH}$ but only at $2 \sigma$ if the true hierarchy is $\mathrm{IH}$. Addition of $\mathrm{T} 2 \mathrm{~K}(5 \nu+5 \bar{\nu})$ and $\mathrm{NO} \nu \mathrm{A}(5 \nu+5 \bar{\nu})$ runs leads only to a marginal improvement but not $3 \sigma$ discrimination. In order to consider a $5 \sigma$ discrimination against vacuum oscillations, we did a simulation of DUNE $(5 \nu+5 \bar{\nu})$ run. Here again, we find that a $5 \sigma$ discrimination is possible only if the true hierarchy is $\mathrm{NH}$ but not if the true hierarchy is $\mathrm{IH}$. However, a $5 \sigma$ discrimination is possible for both hierarchies if the data of DUNE $(5 \nu+5 \bar{\nu})$ run is considered in conjunction with $\mathrm{T} 2 \mathrm{~K}(5 \nu+5 \bar{\nu})$ and $\mathrm{NO} \nu \mathrm{A}(5 \nu+5 \bar{\nu})$ runs, as illustrated in fig. 6. We also note from this figure that values of $q$ out side the range $(1 \pm 0.4)$ are ruled out at $3 \sigma$ or better. 


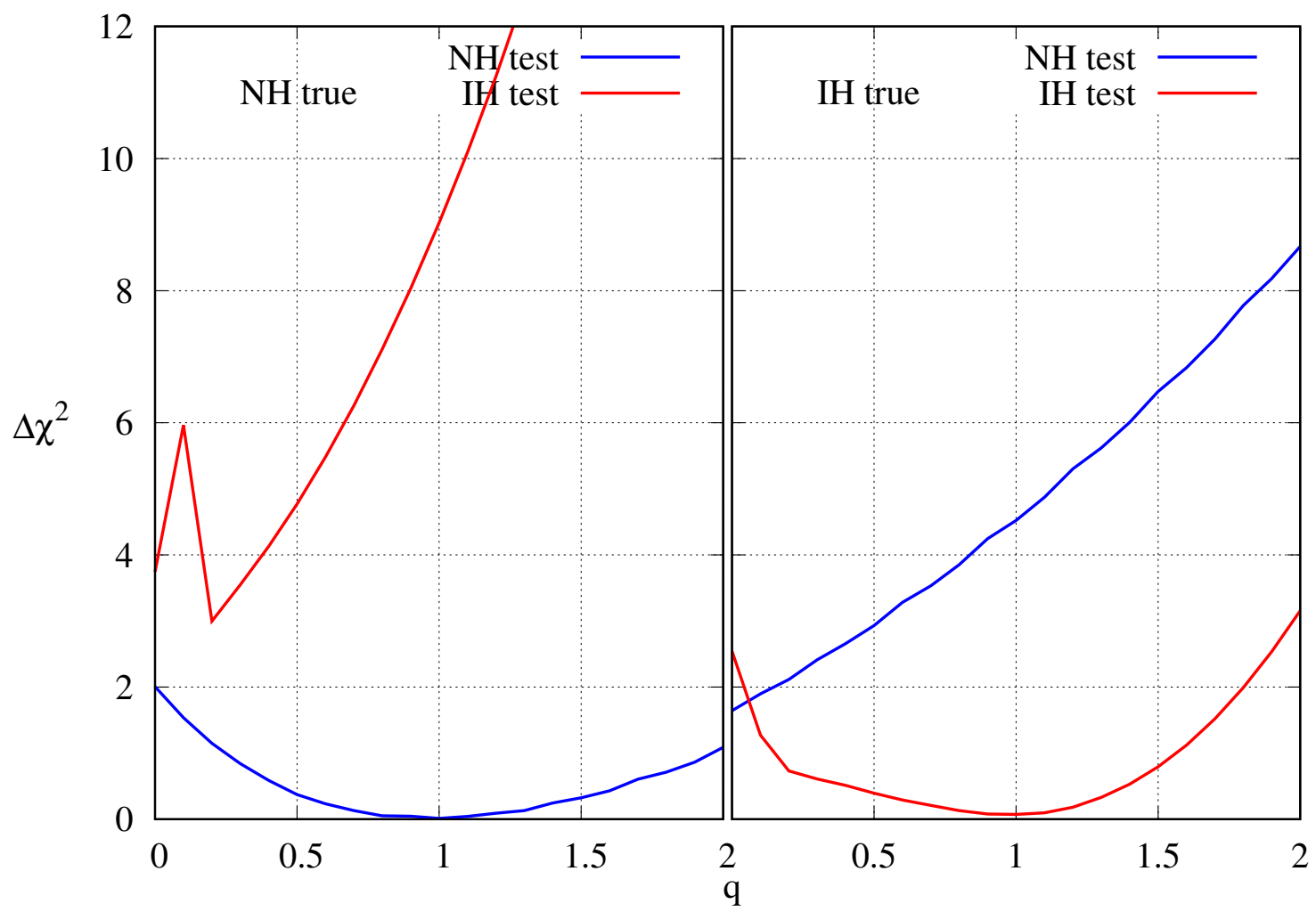

FIG. 4: $\Delta \chi^{2}$ vs $q$ for an expected T2K run with $37.4 \times 10^{20}$ POT each in both $\nu$ and $\bar{\nu}$ mode and a $\mathrm{NO} \nu \mathrm{A}$ run with $30.25 \times 10^{20} \mathrm{POT}$ each in both $\nu$ and $\bar{\nu}$ mode, equivalent to $(5 \nu+5 \bar{\nu})$ runs for each experiment. The left (right) panel assumes the true hierarchy to be normal (inverted). The blue (red) curves assume the test hierarchy to be normal (inverted). Note that data of $\mathrm{T} 2 \mathrm{~K}+\mathrm{NO} \nu \mathrm{A}$ has no ability to discriminate against vacuum oscillations even with such long exposure.

\section{CONCLUSIONS}

Matter effects allow us to determine the sign of neutrino mass-squared differences. The existence of matter effects at the scale of $\Delta_{21}$ is well established [25]. However, at the scale of $\Delta_{31}$, vacuum oscillations fit the data nearly as well as matter modified oscillations. This is true for both atmospheric neutrino data [10] and for present long-baseline accelerator data, as demonstrated in this work. We also show that even extended runs of T2K and $\mathrm{NO} \nu \mathrm{A}$ have no discriminating ability against vacuum oscillations. A $3 \sigma$ discrimination against vacuum oscillations can be achieved with one year neutrino run of DUNE, if NH is the true hierarchy but not if $\mathrm{IH}$ is the true hierarchy. Ruling out vacuum oscillations at $5 \sigma$ 


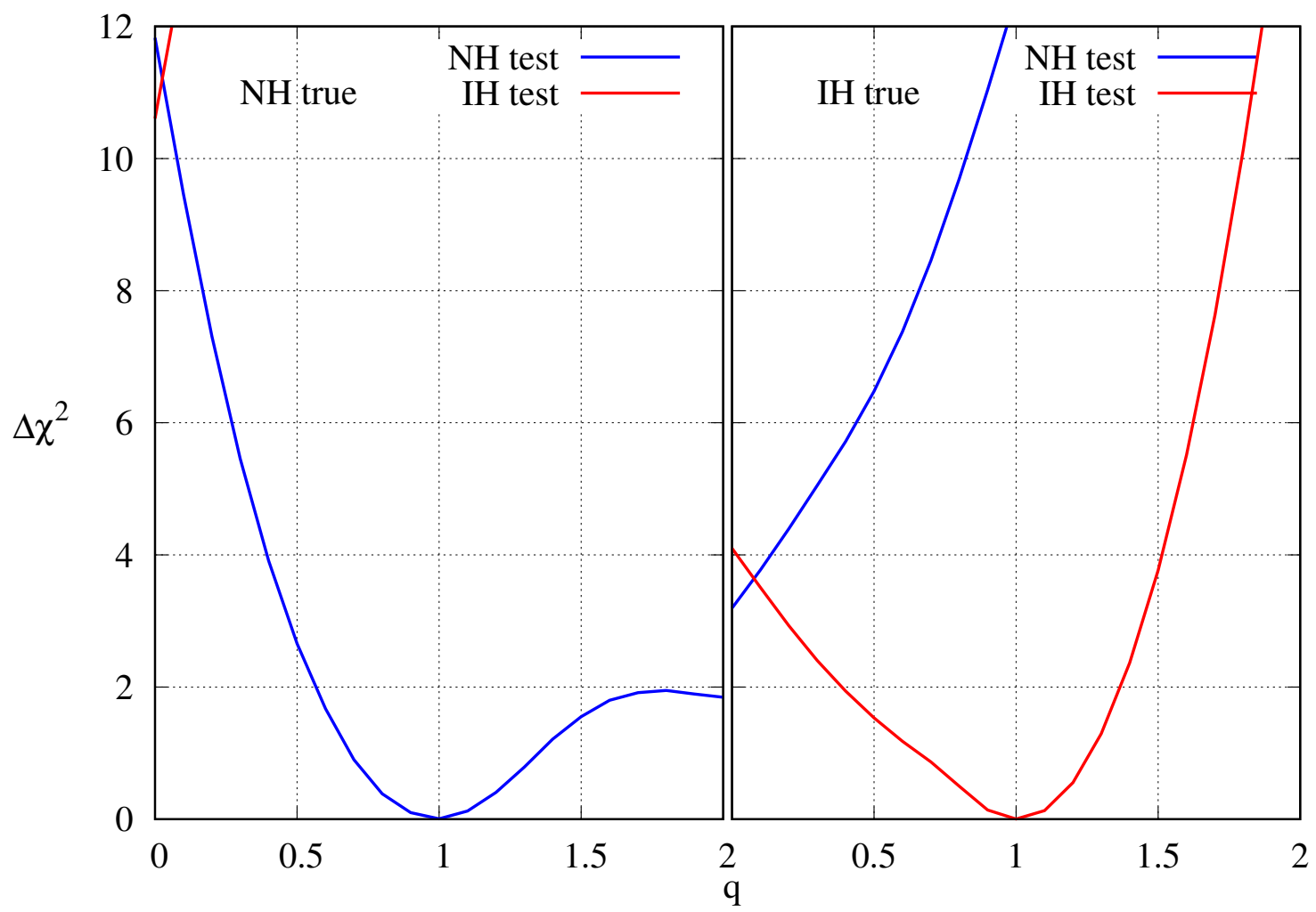

FIG. 5: $\Delta \chi^{2}$ vs $q$ for an expected one year neutrino run of DUNE $\left(14.7 \times 10^{20}\right.$ POT). The left (right) panel assumes the true hierarchy to be normal (inverted). The blue (red) curves assume the test hierarchy to be normal (inverted).

requires the combined data of $(5 \nu+5 \bar{\nu})$ runs of T2K, NO $\nu$ A and DUNE. Such a data can also establish the strength of matter effects with good precision.

\section{ACKNOWLEDGEMENTS}

SB thanks IIT Bombay for financial support.

[1] D. Casper et al., Phys. Rev. Lett. 66, 2561 (1991).

[2] R. Becker-Szendy et al., Phys. Rev. D 46, 3720 (1992).

[3] K. Hirata et al. (Kamiokande-II), Phys. Lett. B 280, 146 (1992). 


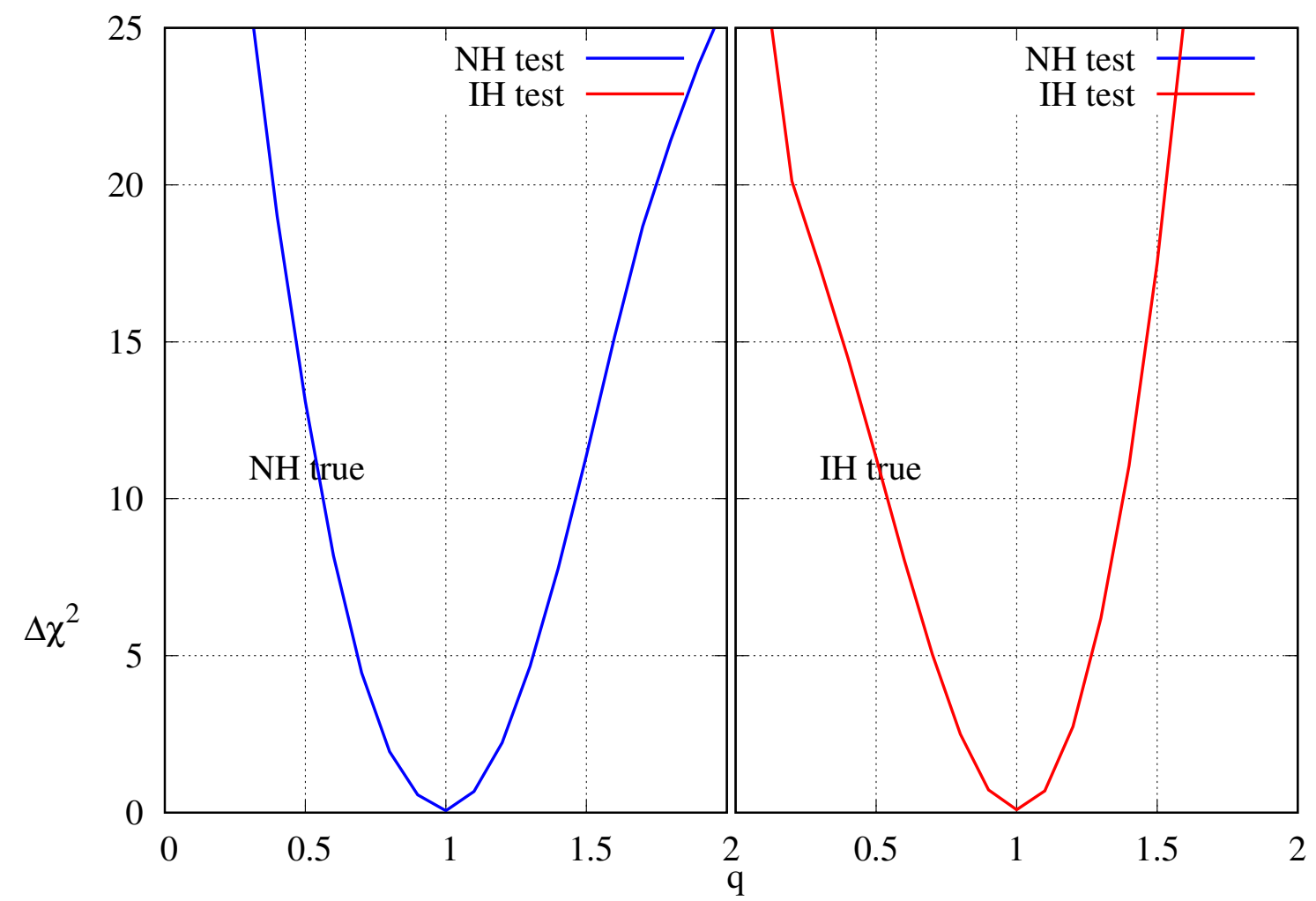

FIG. 6: $\Delta \chi^{2}$ vs $q$ for an expected $(5 \nu+5 \bar{\nu})$ run of DUNE plus equal $\nu$ and $\bar{\nu}$ runs of T2K with $37.4 \times 10^{20} \mathrm{POT}$ and of $\mathrm{NO} \nu \mathrm{A}$ with $30.25 \times 10^{20} \mathrm{POT}$. The left (right) panel assumes the true hierarchy to be normal (inverted). The blue (red) curves assume the test hierarchy to be normal (inverted).

[4] Y. Fukuda et al. (Kamiokande), Phys. Lett. B 335, 237 (1994).

[5] Y. Fukuda et al. (Super-Kamiokande), Phys. Rev. Lett. 81, 1562 (1998), hep-ex/9807003.

[6] D. G. Michael et al. (MINOS), Phys. Rev. Lett. 97, 191801 (2006), hep-ex/0607088.

[7] K. Abe et al. (T2K), Phys. Rev. Lett. 111, 211803 (2013), 1308.0465.

[8] P. Adamson et al. (NOvA), Phys. Rev. Lett. 118, 151802 (2017), 1701.05891.

[9] R. Gandhi, P. Ghoshal, S. Goswami, P. Mehta, S. Uma Sankar, and S. Shalgar, Phys. Rev. D 76, 073012 (2007), 0707.1723.

[10] K. Abe et al. (Super-Kamiokande), Phys. Rev. D 97, 072001 (2018), 1710.09126.

[11] S. Petcov and T. Schwetz, Nucl. Phys. B 740, 1 (2006), hep-ph/0511277.

[12] A. Ghosh and S. Choubey, JHEP 10, 174 (2013), 1306.1423. 
[13] A. Ajmi, A. Dev, M. Nizam, N. Nayak, and S. Uma Sankar, J. Phys. Conf. Ser. 888, 012151 (2017), 1510.02350 .

[14] K. Abe et al. (T2K), Nucl. Instrum. Meth. A 659, 106 (2011), 1106.1238.

[15] D. Ayres et al. (NOvA) (2007).

[16] P. Lipari, Phys. Rev. D 61, 113004 (2000), hep-ph/9903481.

[17] M. Narayan and S. Uma Sankar, Phys. Rev. D 61, 013003 (2000), hep-ph/9904302.

[18] O. Mena and S. J. Parke, Phys. Rev. D70, 093011 (2004), hep-ph/0408070.

[19] S. Prakash, S. K. Raut, and S. U. Sankar, Phys.Rev. D86, 033012 (2012), 1201.6485.

[20] S. Bharti, S. Prakash, U. Rahaman, and S. Uma Sankar, JHEP 09, 036 (2018), 1805.10182.

[21] L. Wolfenstein, Phys. Rev. D17, 2369 (1978).

[22] S. P. Mikheev and A. Yu. Smirnov, Sov. J. Nucl. Phys. 42, 913 (1985), [Yad. Fiz.42,1441(1985)].

[23] S. P. Mikheev and A. Yu. Smirnov, Nuovo Cim. C9, 17 (1986).

[24] J. N. Bahcall, M. C. Gonzalez-Garcia, and C. Pena-Garay, JHEP 08, 016 (2004), hep$\mathrm{ph} / 0406294$.

[25] G. Fogli, E. Lisi, A. Marrone, and A. Palazzo, Prog. Part. Nucl. Phys. 57, 742 (2006), hep$\mathrm{ph} / 0506083$.

[26] A. Cervera, A. Donini, M. B. Gavela, J. J. Gomez Cadenas, P. Hernandez, O. Mena, and S. Rigolin, Nucl. Phys. B579, 17 (2000), [Erratum: Nucl. Phys.B593,731(2001)], hep$\mathrm{ph} / 0002108$.

[27] M. Freund, Phys.Rev. D64, 053003 (2001), hep-ph/0103300.

[28] P. Huber, M. Lindner, and W. Winter, Comput.Phys.Commun. 167, 195 (2005), hep$\mathrm{ph} / 0407333$.

[29] P. Huber, J. Kopp, M. Lindner, M. Rolinec, and W. Winter, Comput.Phys.Commun. 177, 432 (2007), hep-ph/0701187.

[30] I. Esteban, M. C. Gonzalez-Garcia, A. Hernandez-Cabezudo, M. Maltoni, and T. Schwetz, JHEP 01, 106 (2019), 1811.05487.

[31] http://www.nu-fit.org/?q=node/45.

[32] M. Acero et al. (NOvA), Phys. Rev. Lett. 123, 151803 (2019), 1906.04907.

[33] K. Abe et al. (T2K), Nature 580, 339 (2020), [Erratum: Nature 583, E16 (2020)], 1910.03887.

[34] K. Abe et al. (T2K), Phys. Rev. Lett. 121, 171802 (2018), 1807.07891. 
[35] B. Abi et al. (DUNE) (2018), 1807.10334.

[36] B. Abi et al. (DUNE) (2018), 1807.10327.

[37] B. Abi et al. (DUNE) (2018), 1807.10340. 